\title{
Multicentric Adrenal Myelolipomas: Case Report
}

\author{
Kiyasettin Asil ${ }^{\mathrm{a}, \mathrm{b}}$, Mehmet Ersavas ${ }^{\mathrm{a}}$, Yakup Ersel Aksoy ${ }^{\mathrm{a}}$
}

\begin{abstract}
Adrenal myelolipomas are rare and non-functioning benign tumors. They are composed of adipose tissue and hemopoietic elements in varying proportions. In this report we present the case with two myelolipomas at the right adrenal gland that was undefined in the literature. A 40-year-old female patient referred our hospital for overweight. During searching the etiology of obesity, lesions were found incidentally on the abdominal computed tomography (CT) imaging. On the right adrenal gland two well-circumscribed mass lesions were found, and magnetic resonance imaging (MRI) was performed. After intravenous (IV) gadolinium administration no contrast enhancement was seen. Surgical operation was recommended to the patient, but the patient has not accepted yet. In the literature, bilateral adrenal myelolipomas were reported, but multicentric adrenal myelolipomas in the same adrenal gland have not been reported yet. In our case we have shown multicentric adrenal myelolipomas which can be seen in the same adrenal gland. Radiologic imaging keeps the most important place in the diagnosis of lesion and planning of the treatment.
\end{abstract}

Keywords: Multicentric; Adrenal; Myelolipoma

\section{Introduction}

Adrenal myelolipomas are rare and non-functioning benign tumors [1]. They are composed of adipose tissue and hemopoietic elements in varying proportions $[1,2]$. Even though adrenal myelolipomas are usually associated with obesity, hypertension and diabetes mellitus, generally are asymptomatic and discovered incidentally at autopsy or by radiologic imaging [2]. Lesions are usually unilateral, but it has been rarely reported on both sides in the literature $[3,4]$. In this report we

\section{Manuscript accepted for publication August 07, 2014}

aDepartment of Radiology, Sakarya University Faculty of Medicine, 54100, Korucuk, Sakarya, Turkey

bCorresponding Author: Kiyasettin Asil, Department of Radiology, Sakarya University Faculty of Medicine, 54100, Korucuk, Sakarya, Turkey.

Email: drkiyasettin@gmail.com

doi: http://dx.doi.org/10.14740/jmc1917w present the case with two myelolipomas at the right adrenal gland that was undefined in the literature.

\section{Case Report}

In our case, the patient was a 40-year-old female and referred our hospital for overweight. Her height was $156 \mathrm{~cm}$ and weight was $76 \mathrm{~kg}$ and body mass index (BMI) was $31 \mathrm{~kg} / \mathrm{m}^{2}$, and according to actual BMI classification she was obese [5]. During searching the etiology of obesity, lesions were found incidentally on the abdominal computed tomography (CT) imaging. On the right adrenal gland well circumscribed two mass lesions were found on the CT that were $71 \times 69 \times 75 \mathrm{~mm}$ and $59 \times 48 \times 65 \mathrm{~mm}$ in size, and their average density was -70 Hounsfield unit (HU) (Fig. 1, 2). On the wall of the mass lesions, calcifications were seen. On the T1-weighted and T2weighted magnetic resonance imaging (MRI) sequence, two hyperintense lesions including hypointense areas were seen. On the fat-suppressed MRI sequence signal loss was more ap-

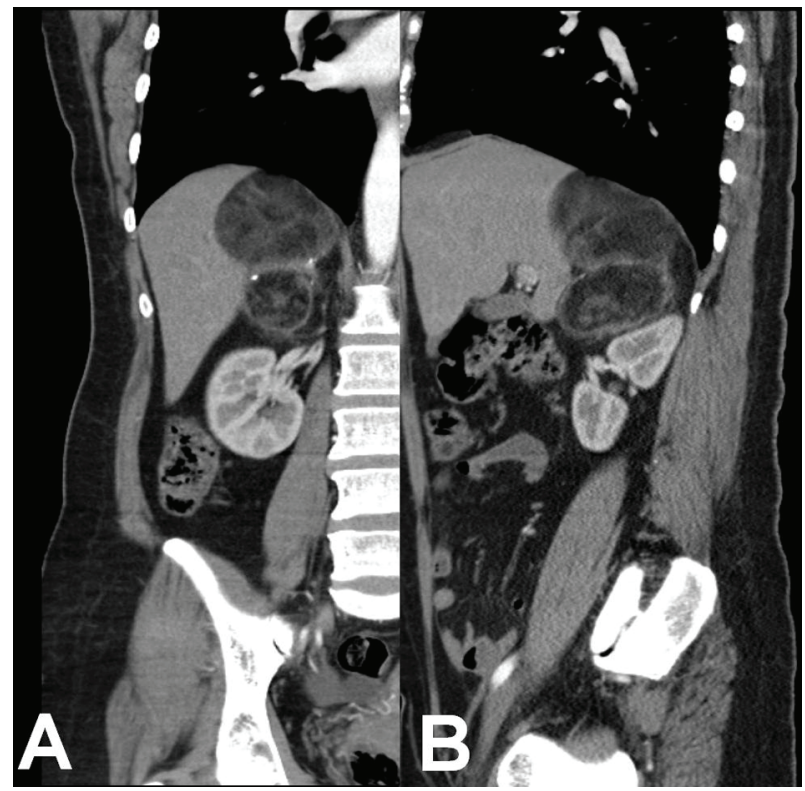

Figure 1. Coronal (A) and sagittal (B) CT images show two separate adrenal fatty masses. Calcifications are seen in the wall of masses. 


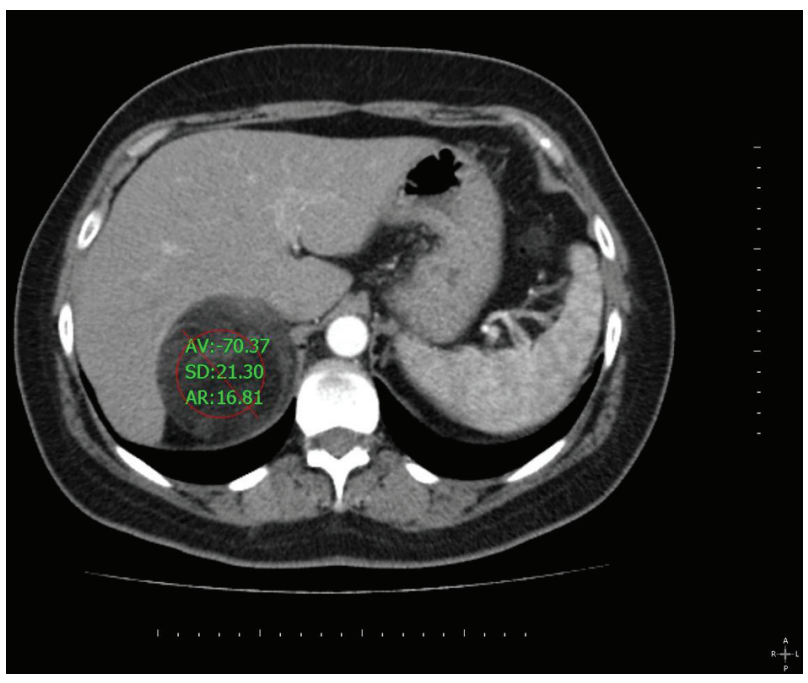

Figure 2. Axial CT image shows well-defined mass in the right adrenal gland with adipose tissue density (average $-70 \mathrm{HU}$ ).

parent than out-of-phase MRI sequence signal loss (Fig. 3). After intravenous (IV) gadolinium administration no contrast enhancement was seen (Fig. 4). Biochemical parameters of the patient were in normal limits. Catecholamines were normal in $24 \mathrm{~h}$ urine collection examination. Surgical operation was recommended to the patient, but the patient has not accepted yet.

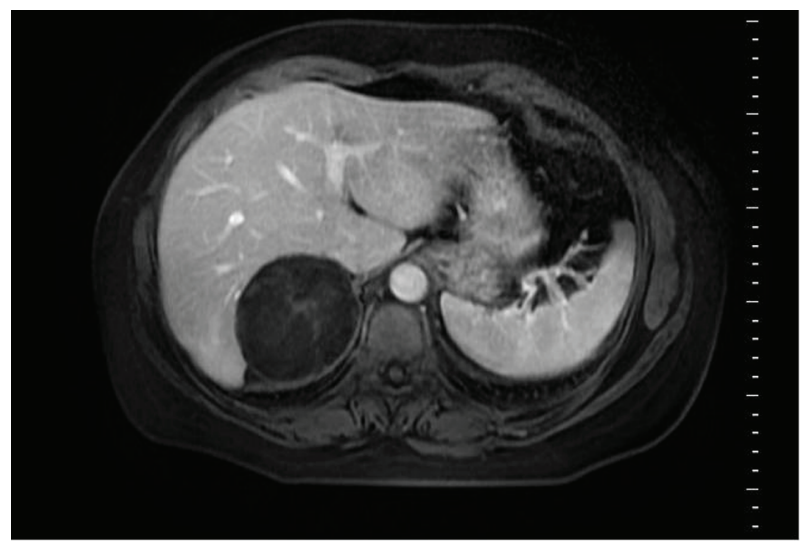

Figure 4. No contrast enhancement is seen on post-contrast MRI image.

\section{Discussion}

Adrenal myelolipoma was first described by Gierke in 1905, and the term myelolipoma was coined by Oberling in 1929 [4]. They are infrequent, slow growing, benign, usually unilateral and less than $4 \mathrm{~cm}$ in diameter $[2,4,6]$. Adrenal myelolipomas generally occur from fifth to seventh decade of life, and there is no gender preference [7]. They are non-functional tumors composed of mature adipocytes and active hematopoietic ele-

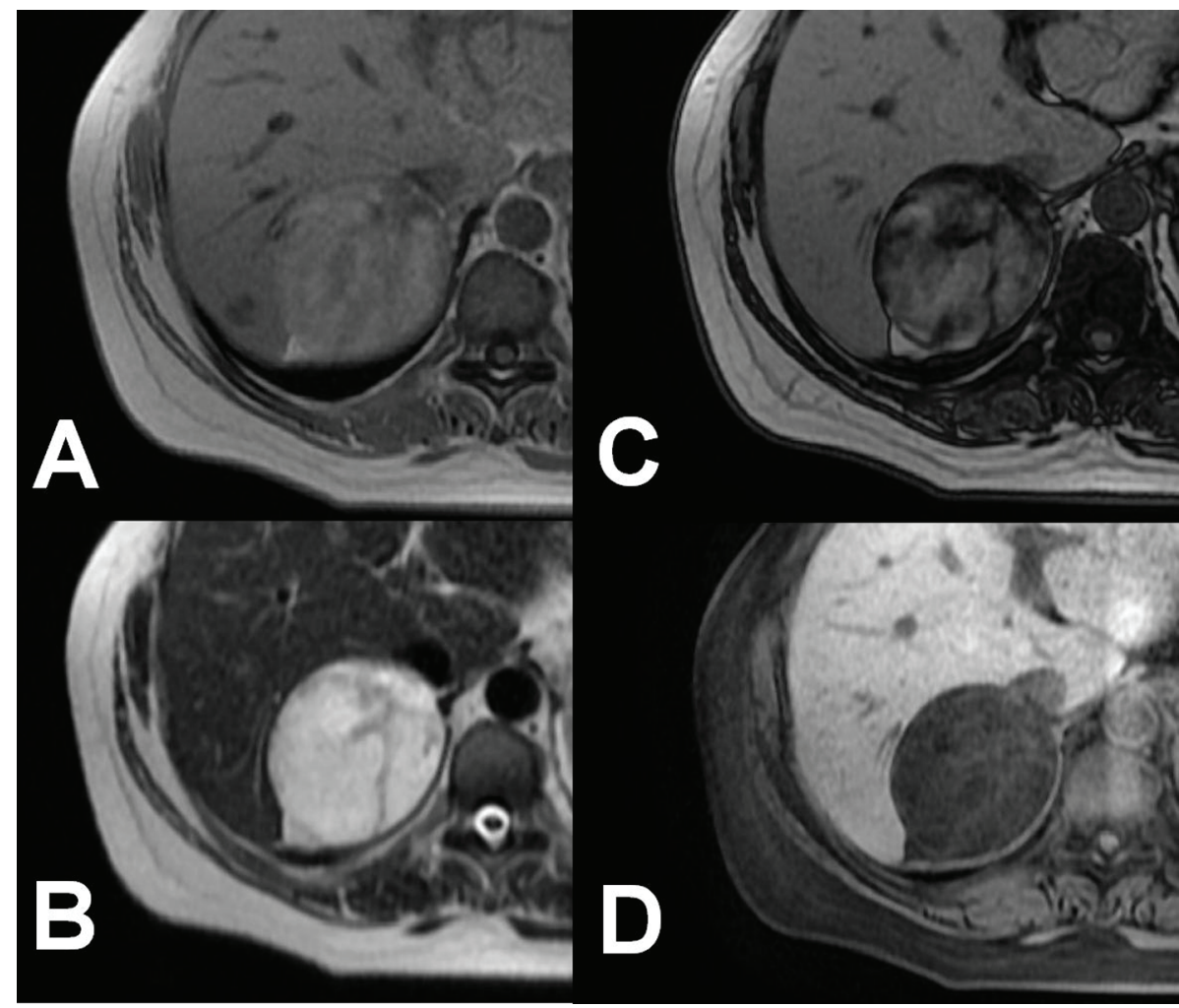

Figure 3. Right adrenal mass on MRI: on axial T1-weighted in-phase image (A) lesion is heterogenous hyperintense, on T2weighted image (B) hyperintense and on T1-weighted out-of-phase image (C) signal void areas are seen and on fat-suppressed image (D) the signal of the mass is suppressed almost totally. 
ments and take origin from the adrenal cortex [2, 3]. Although there are a few theories on AL histogenesis, it is still uncertain [3]. The most accepted theory is metaplasia of reticuloendothelial cells of blood capillaries in the adrenal gland in response to stimuli such as chronic infection, stress or necrosis [2]. According to recent studies, they comprise $2.6 \%$ of all primary adrenal masses [2]. When exceeding the diameter of $8 \mathrm{~cm}$, they are called "giant adrenal myelolipomas" [8]. When they are increasing severely in size, tumor compression symptoms may occur [1]. The most common symptoms are abdominal pain, hematuria and abdominal mass $[1,6]$. Abdominal pain occurs probably due to hemorrhage, tumor necrosis or mechanical compression [6]. Adrenal myelolipomas have close associations with obesity, hypertension, chronic disease and malignancies [4]. As we know that there is a close relationship between adrenal myelolipoma and obesity and patients are usually asymptomatic, our patient was also obese and asymptomatic too.

Adrenal myelolipomas are mostly discovered by imaging for unrelated reasons, and diagnosis of them is based on radiologic imaging $[4,6]$. Adrenal myelolipomas are seen as lucent areas on plain film and as hyperechoic mass in the adrenal region on ultrasonography [1]. Depending on myeloid tissue component of the tumor, the mass may contain hypoechoic area on the ultrasound [6]. CT is most sensitive dedication imaging modality for diagnosing them [6]. CT shows fat density ( $-30 \mathrm{HU}$ to $-100 \mathrm{HU})$ in the adrenal tumor and adrenal myelolipomas are avascular at angiography $[2,7]$. MRI shows characteristic hyperintense mass due to fat component on T1-weighted and T2-weighted sequences. Their signals are suppressed on fat suppression imaging, but persistent high signal intensity areas may be seen as a result of myeloid tissue component or hemorrhage [6]. These signal changes occur depending on the fat content of the tumor [6]. In out-of-phase sequences, the microscopic fat loses signal, because fat and water protons in the same voxel equalize each other. So as in an adrenal myelolipoma, clear fat saturation sequence loses more signal than chemical shift imaging, because including macroscopic fat is typical character of a myelolipoma. Unlike a lipid-rich adrenal adenoma loses greater signal intensity on chemical shift imaging because of comprising microscopic fat [2]. In T1-weighted sequences, myeloid tissue component of the adrenal lipomas shows low signal intensity and in T2weighted sequences shows mild signal intensity [4]. In around $27 \%$ of cases, calcifications can be observed [2]. In our case, two adrenal myelolipomas which have fat density on CT were seen in right surrenal gland and also peripheral calcifications are seen. On the patient's T1-weighted and T2-weighted MRI sequences, typical hyperintensity was determined and on the fat-suppressed MRI sequences, signal loss was explicit.

Retroperitoneal lipoma or liposarcoma, upper pole renal angiomyolipoma and retroperitoneal teratoma have to be considered in the differential diagnosis of the adrenal myelolipoma [2].

The treatment of adrenal myelolipomas should be individualized [9]. The patient has a small, less than $5 \mathrm{~cm}$ in diameter, and asymptomatic myelolipoma, that could be observed conservatively around $6-12$ months interval follow-up by using ultrasound or CT for a 1 - 2 year period $[2,9]$. On the contrary, a symptomatic lesion or a large than $5 \mathrm{~cm}$ in diameter myelolipoma should be surgically excised, because there are reports about spontaneous rupture and hemorrhage risks of the mass leading to life-threatening cardiovascular shock [9]. The classical treatment of both functioning and non-functioning adrenal tumors has become laparoscopic adrenalectomy by starting to do minimally invasive surgery [2]. When compared with open surgery, acceptable decrease in the peri-operative morbidity, hospitalization and shorter convalescence are seen with laparoscopic procedure [2]. Unfortunately, this procedure is not applied for masses bigger than $10 \mathrm{~cm}$ or with adhesions and infiltration of the surrounding structures [6]. In the case of bilateral adrenal myelolipomas, a staged tumor removal is preferable, removing the larger one and continuing to observe the counterside myelolipoma as long as possible in an effort to avoid adrenal insufficiency and a lifetime of steroid replacement [4].

However being usually asymptomatic and unilateral, due to widened size adrenal myelolipomas can make patients symptomatic. In the literature, bilateral adrenal myelolipomas were reported, but multicentric adrenal myelolipomas in the same adrenal gland have not been reported yet. In our case we have shown multicentric adrenal myelolipomas which can be seen in the same adrenal gland. Radiologic imaging keeps the most important place in the diagnosis of lesion and planning of the treatment.

\section{Grant Support}

No.

\section{Conflict of Interest}

No.

\section{References}

1. Mukherjee S, Pericleous S, Hutchins RR, Freedman PS. Asymptomatic giant adrenal myelolipoma. Urol J. 2010;7(1):66-68.

2. Bano S, Yadav SN, Chaudhary V, Garga UC. Symptomatic giant adrenal myelolipoma associated with cholelithiasis: Two case reports. Urol Ann. 2012;4(1):55-60.

3. Brogna A, Scalisi G, Ferrara R, Bucceri AM. Giant secreting adrenal myelolipoma in a man: a case report. J Med Case Rep. 2011;5:298.

4. Cha JS, Shin YS, Kim MK, Kim HJ. Myelolipomas of both adrenal glands. Korean J Urol. 2011;52(8):582-585.

5. Switzer NJ, Mangat HS, Karmali S. Current trends in obesity: body composition assessment, weight regulation, and emerging techniques in managing severe obesity. J Interv Gastroenterol. 2013;3(1):34-36.

6. Wani NA, Kosar T, Rawa IA, Qayum A. Giant adrenal myelolipoma: Incidentaloma with a rare incidental asso- 
ciation. Urol Ann. 2010;2(3):130-133.

7. Chakrabarti I, Ghosh N, Das V. Giant adrenal myelolipoma with hemorrhage masquerading as retroperitoneal sarcoma. J Midlife Health. 2012;3(1):42-44.

8. Kapetanakis S, Drygiannakis I, Tzortzinis A, Papanas N, Fiska A. A giant adrenal lipoma presenting in a woman with chronic mild postprandial abdominal pain: a case report. J Med Case Rep. 2011;5:136.

9. Tyritzis SI, Adamakis I, Migdalis V, Vlachodimitropoulos D, Constantinides CA. Giant adrenal myelolipoma, a rare urological issue with increasing incidence: a case report. Cases J. 2009;2:8863. 